\title{
Role of Whole-Spine Screening Magnetic Resonance Imaging Using Short Tau Inversion Recovery or Fat- Suppressed T2 Fast Spin Echo Sequences for Detecting Noncontiguous Multiple-Level Spinal Tuberculosis
}

\author{
${ }^{1}$ Department of Radiology, Saint Louis University Hospital, St. Louis, MO, USA \\ ${ }^{2}$ School of Medicine, Jawaharlal Nehru Medical College, Aligarh, India \\ ${ }^{3}$ Department of Ophtalmology, Jawaharlal Nehru Medical College, Aligarh, India \\ ${ }^{4}$ Department of Orthopedic Surgery, Jawaharlal Nehru Medical College, Aligarh, India \\ ${ }^{5}$ Department of Pediatrics, Jawaharlal Nehru Medical College, Aligarh, India
}

Mohammed Azfar Siddiqui ${ }^{1}$, Sara Sartaj ${ }^{2}$, Syed Wajahat A. Rizvi ${ }^{3}$, Mohammad Jesan Khan ${ }^{4}$, Iraj Alam Khan ${ }^{5}$

Study Design: Retrospective review.

Purpose: The purpose of the present study was to evaluate the role of whole-spine screening using short tau inversion recovery (STIR) or fat-suppressed T2W fast spin echo (FSE) sequences in patients with spinal tuberculosis (TB).

Overview of Literature: The identification of noncontiguous multiple-level spinal tuberculosis (NMLST), symptomatic or not, is important because of its management implications. Most centers do not perform routine whole-spine magnetic resonance imaging (MRI), and the reported incidence of NMLST varies from $1.1 \%$ to $74.1 \%$.

Methods: We completed a retrospective review of clinical and radiographic data of 365 patients with spinal TB who presented at Jawaharlal Nehru Medical College, Aligarh over 5 years. The final analysis included 187 patients who full filled the inclusion criteria, consisting of availability of whole-spine MRI and confirmation of vertebral TB. Diagnosis of NMLST was considered when other vertebral lesions were identified in addition to the primary vertebral disease, with the lesions separated by at least one normal spinal segment. The primary site was defined as the site for which the patient had been referred for MRI.

Results: NMLST was identified in 47 of 187 patients investigated using whole-spine MRI. The incidence was $25.1 \%$, which was higher than that in earlier reports where whole-spine MRI was not routinely performed. The lumbar spine was involved in 37 patients, thoracic spine in 25, cervical spine in 16, and sacrum in five patients. Combined lumbar spine and thoracic spine involvement was observed in 19 patients. Thirteen patients had lumbar and cervical spine involvement, nine had thoracic and cervical spine involvement, four had combined lumbar and sacral spine involvement, and the remaining two had thoracic and sacral spine involvement.

Conclusions: Tubercular spondylitis may affect the spine at multiple noncontiguous sites with the majority of additional affected sites remaining asymptomatic. Routine whole-spine MRI using all recommended sequences is not cost-effective and hence not feasible. Therefore, we recommend whole-spine screening using STIR or fat-suppressed T2W FSE sequences in all patients with suspected spinal TB. This screening is cost-effective compared with full-protocol MRI and detects additional cases of NMLST over conventional practice.

Keywords: Tuberculosis; Spine; Magnetic resonance imaging

Received Sep 27, 2017; Revised Nov 1, 2017; Accepted Nov 26, 2017

Corresponding author: Mohammed Azfar Siddiqui

Department of Radiology, Saint Louis University, 374 S. Grand Blvd, St. Louis, MO 63103, USA

Tel: +1-717-649-8167 (M), E-mail: drazfarsiddiqui@gmail.com

*This article was presented at the American Society of Emergency Radiology Annual Scientific Meeting and Postgraduate Course, 9th October-12th October, 2013 at Cambridge (overlooking Boston), Massachusetts, USA. 


\section{Introduction}

Tuberculosis (TB) remains a leading infectious cause of death worldwide [1]. Once on the decline and almost eradicated in industrialized countries, TB is re-emerging as a major public health threat. Skeletal TB accounts for approximately $1 \%-2 \%$ of all infected patients [2]. The spine is the most common site for skeletal TB, accounting for $50 \%$ of cases [3].

Continuous vertebral involvement is the most common disease pattern [4]. Noncontiguous multiple-level spinal tuberculosis (NMLST) is an atypical and rare form of spinal TB. It is defined as vertebral disease in addition to the primary lesion, with diseased spinal segments separated by at least one normal spinal segment. There is large variability in the reported incidence of NMLST, ranging from $1.1 \%$ to $71.4 \%[4,5]$. In the present study, we sought to discuss the role of whole-spine screening using short tau inversion recovery (STIR) or fat-suppressed T2W fast spin echo (FSE) magnetic resonance imaging (MRI) sequences in patients with signs and symptoms suggestive of spinal TB.

\section{Materials and Methods}

\section{Patients}

We completed a retrospective review of clinical and radiographic data that were prospectively collected on 365 patients with spinal TB who were diagnosed and treated at Jawaharlal Nehru Medical College Hospital, Aligarh, from January 2010 to January 2015. The ethical committee of the Jawaharlal Nehru Medical College Hospital approved the study and informed consent was waived. Inclusion criteria included available whole-spine MRI performed on admission and confirmation of vertebral TB by histology, microbiology, or positive response to anti-tubercular treatment.

The primary site was defined as the site for which the patient had been referred for MRI. Diagnosis of NMLST was considered when other vertebral lesions were identified in addition to the primary vertebral disease, with the lesions separated by at least one normal spinal segment.

\section{Diagnosis}

Diagnosis was confirmed after open surgical biopsy or image-guided percutaneous needle biopsy by histology or microbiology. Histological evaluation of biopsy samples was performed to look for granulomas, Langhans giant cells, and caseous necrosis. Microscopic examination was performed to check for acid-fast bacilli. Specimens were cultured for a total of 6 weeks before being declared negative. For patients where a tissue confirmation of TB was not available, a positive response to anti-tubercular treatment was considered diagnostic. The MRI protocol varied among patients but for the most part included the following pulse sequences: $\mathrm{T} 1 \mathrm{~W}, \mathrm{~T} 2 \mathrm{~W}$ in the sagittal plane, STIR in the coronal plane, and axial T1W and T2W. Postcontrast fat-suppressed T1W images were obtained in three orthogonal planes.

\section{Statistics analysis}

The statistical analysis was performed using Microsoft Excel (Microsoft Corp., Redmond, WA, USA) and SPSS software ver. 16.0 (SPSS Inc., Chicago, IL, USA).

\section{Results}

Of 365 patients with spinal TB, only 187 met the inclusion criteria and were included in the final review. This was largely due to the non-availability of whole-spine MRI. The average age was 37 years (range, 7-81 years), with 18 females and 29 males. The incidence of NMLST in patients investigated using whole-spine MRI was 25.1\% (47/187 patients). The remaining 140 patients showed single or contiguous spinal lesions. Their average age was 29 years (range, 4-82 years), with 63 females and 77 males. Concurrent pulmonary TB was identified in 39 of 187 patients. The two groups were not statistically different when compared for age, sex, and outcome.

The diagnosis of TB was made with microbiology in 108 patients and histopathology in 72 patients. In 28 patients, both microbiology and histopathology were available. In 35 patients, a presumptive diagnosis was made based on a positive response to anti-tubercular treatment. MRI revealed at least one typical TB lesion in all $187 \mathrm{pa}$ tients. These included intervertebral disc narrowing, T2 hyperintensity and heterogeneous pattern of contrast enhancement of involved vertebral bodies, posterior element involvement, subligamentous spread, epidural extension, and presence of paraspinal or intraosseous abscess (Figs. 1, 2). Of the 47 patients with multiple level involvement, all 


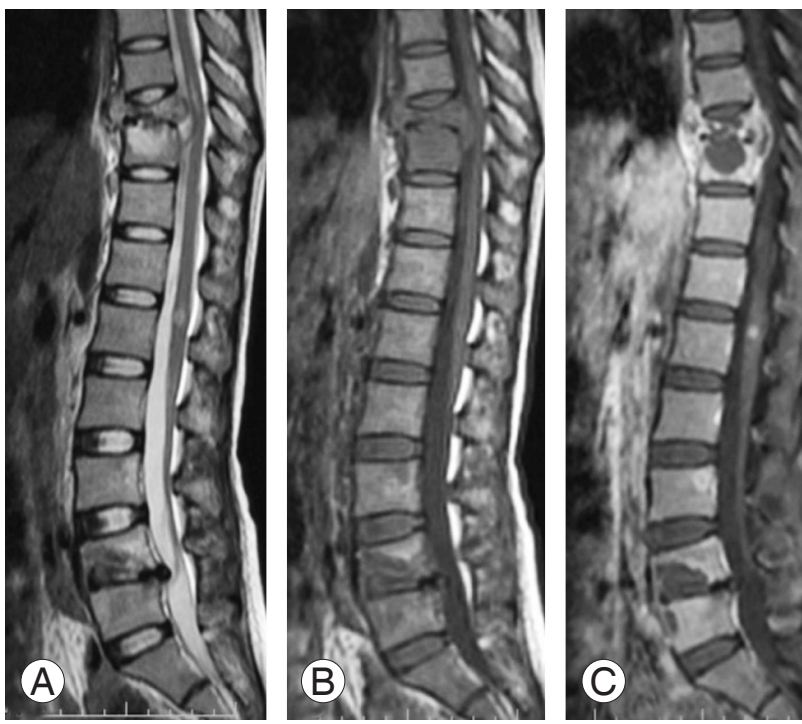

Fig. 1. Sagittal T2W (A), T1W (B), and post-contrast fat-suppressed T1W (C) sequences reveal noncontiguous multiple-level spinal tuberculosis involvement at T9-T10 and L4-L5 levels. Additional enhancing foci of infection were seen in L3 vertebra and conus medullaris.
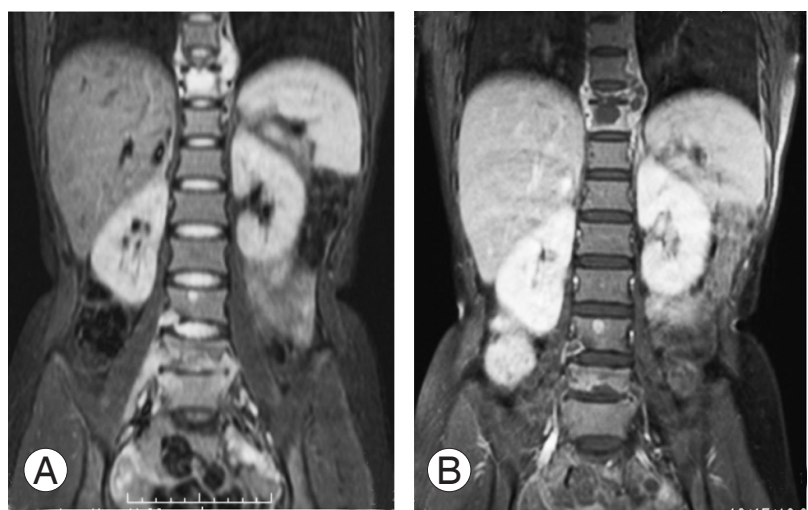

Fig. 2. Coronal short tau inversion recovery (A) and post-contrast fatsuppressed T1W (B) sequences reveal noncontiguous multiple-level spinal tuberculosis involvement at T9-T10 and L4-L5 levels. Para spinal soft tissue and $\mathrm{L} 3$ vertebral involvement was also observed.

had clear evidence of noncontiguous lesions on MRI.

With regard to the distribution of lesions, 37 patients had two noncontiguous foci involved, while the remaining 10 patients had three or more discrete lesions. The distribution of involved vertebrae showed a predilection for the lower thoracic and lumbosacral vertebrae, in keeping with distribution patterns described by Hodgson. The lumbar spine was involved in 37 patients, thoracic spine in 25 , cervical spine in 16, and sacrum in five. Combined lumbar spine and thoracic spine involvement was observed in
Table 1. Distribution of lesions

\begin{tabular}{lc} 
Region & No. of patients \\
\hline Lumbar spine & 37 \\
\hline Thoracic spine & 25 \\
\hline Cervical spine & 16 \\
\hline Sacrum & 5 \\
\hline Lumbar spine and thoracic spine & 19 \\
\hline Lumbar and cervical spine & 13 \\
\hline Thoracic and cervical spine & 9 \\
\hline Lumbar and sacral spine & 4 \\
\hline Thoracic and sacral spine & 2 \\
\hline
\end{tabular}

19 patients, lumbar and cervical spine involvement in 13, thoracic and cervical spine involvement in nine, and combined lumbar and sacral spine involvement in four, while the remaining two patients showed thoracic and sacral spine involvement (Table 1).

\section{Discussion}

TB is the leading infectious cause of mortality and morbidity in developing and third world countries. TB is making a dramatic comeback in industrialized countries as a result of increased immigration, acquired immunodeficiency syndrome, and new drug-resistant strains [6]. The spine is the most common site of osseous TB due to the hematogenous spread of the infection [7]. The other mechanisms underlying spinal TB involvement include venous seeding, lymphatic spread, and direct invasion from adjacent viscera or lymph nodes. The infection typically starts in the anterior aspect of the vertebral body adjacent to the intervertebral disc and then spreads to the vertebral bodies under the longitudinal ligaments involving multiple adjacent vertebrae [6,8-10].

NMLST due to the involvement of remote vertebrae is uncommon. The proposed mechanism is through the valveless vertebral venous system, which allows the bacilli to travel to new vertebrae and establish noncontiguous lesions without being filtered through the lungs or lymph nodes. Another explanation is the preferential hematogenous seeding of different spinal segments, but this explanation is less accepted [6].

The identification of NMLST, symptomatic or not, is important because it influences the treatment approach [4]. Anti-tubercular chemotherapy is considered the 
mainstay of treatment for uncomplicated spinal TB. It also improves surgical outcomes by promoting healing and reducing recurrence [11].

There is a paucity of information regarding the treatment of NMLST. However, the presence of noncontiguous lesions influences decisions surrounding surgical instrumentation and the number of levels instrumented. In addition, if a level is not surgically treated, there may be a need for bracing. The aim of our study was to identify unsuspected sites of disease so that these sites could be monitored and supportive measures could be directed to these sites, thereby potentially preventing pathological fractures or the need for surgery.

Due to the rarity of NMLST, our literature review yielded mostly case reports. The reported incidence of NMLST is highly variable, ranging from $1.1 \%$ to $71.4 \%[4,5]$. This is likely due to the different types of imaging modalities used, including radiography, computed tomography (CT), bone scintigraphy, and/or MRI. The centers that do not routinely perform whole-spine MRI reported a lower incidence. Kaila et al. [4] reported a very high incidence of $71.4 \%$ but admitted that their data likely overestimated the true incidence of NMLST due to the small number of cases presented. Their population could have also been affected by selection bias as their center is a regional referral center for spinal deformities [4]. In our study, the incidence of NMLST using whole-spine MRI was $25.1 \%$. The thoracic and lumbar spine were most commonly affected, similar to previous reports.

MRI is the imaging technique of choice in spinal infections due to its high sensitivity and specificity [12]. Given its superior contrast resolution, it is useful for identifying contiguous and noncontiguous vertebral involvement. MRI also provides critical information about spinal cord involvement and the extent of epidural extension. CT is less accurate for defining epidural extension but is useful in assessing bone destruction. Bone scan findings are nonspecific, and radiographs are insensitive in patients with early disease.

The reason for the increase in the reported incidence of noncontiguous involvement is not fully understood, but it likely relates to the increased use of whole-spine MRI, as in our study. Although it is not always possible and also not necessary to routinely use all sequences of wholespine MRI, we strongly recommend whole-spine screening using STIR or fat-suppressed T2W FSE sequences in all patients with signs or symptoms suggestive of spinal
TB. The cost-benefit ratio is good, as our recommendation is not to perform whole-spine MRI utilizing all the sequences but just to screen the spine in a single sagittal plane utilizing a single T2W or STIR sequence. These are FSE sequences and therefore will not substantially increase scan time. The patient turnover time is not increased; therefore, the cost of the MRI should remain unchanged as well.

\section{Conclusions}

The identified incidence of NMLST was $25.1 \%$ in our study. The prevalence of NMLST in other populations may not be the same as that revealed in our study group given the wide disparity in disease prevalence between developing and developed countries. However, there is a trend toward a higher reported incidence of NMLST compared with continuous vertebral involvement. This trend may not necessarily reflect increasing incidence of disease but instead may reflect increased detection due to the more widespread use of MRI. MRI scanning is much more sensitive than plain radiography for detecting NMLST. The identification of NMLST is important because a large proportion of the affected noncontiguous sites may be asymptomatic. The identification of such asymptomatic sites can alter the therapeutic strategy and thereby limit the morbidity caused by TB. We strongly recommend that the entire spine be screened in patients with TB using STIR or fat-suppressed T2W FSE MRI sequences.

\section{Conflict of Interest}

No potential conflict of interest relevant to this article was reported.

\section{References}

1. Reichman LB. Tuberculosis elimination: what's to stop us? Int J Tuberc Lung Dis 1997;1:3-11.

2. Chapman M, Murray RO, Stoker DJ. Tuberculosis of the bones and joints. Semin Roentgenol 1979;14:26682.

3. Siddiqui MA, Ali Rizvi SW, Ali Rizvi SA. Isolated tubercular scaphoid osteomyelitis: a case report. Orthop Surg 2012;4:64-6.

4. Kaila R, Malhi AM, Mahmood B, Saifuddin A. The incidence of multiple level noncontiguous vertebral 
tuberculosis detected using whole spine MRI. J Spinal Disord Tech 2007;20:78-81.

5. Yalniz E, Pekindil G, Aktas S. Atypical tuberculosis of the spine. Yonsei Med J 2000;41:657-61.

6. Polley P, Dunn R. Noncontiguous spinal tuberculosis: incidence and management. Eur Spine J 2009;18:1096-101.

7. Garg RK, Somvanshi DS. Spinal tuberculosis: a review. J Spinal Cord Med 2011;34:440-54.

8. Turgut M. Multifocal extensive spinal tuberculosis (Pott's disease) involving cervical, thoracic and lumbar vertebrae. Br J Neurosurg 2001;15:142-6.

9. Griffith JF, Kumta SM, Leung PC, Cheng JC, Chow LT, Metreweli C. Imaging of musculoskeletal tuberculosis: a new look at an old disease. Clin Orthop
Relat Res 2002;(398):32-9.

10. Pande KC, Babhulkar SS. Atypical spinal tuberculosis. Clin Orthop Relat Res 2002;(398):67-74.

11. Huang J, Zhang H, Zeng K, Gao Q. The clinical outcomes of surgical treatment of noncontiguous spinal tuberculosis: a retrospective study in 23 cases. PLoS One 2014;9:e93648.

12. Khalid M, Siddiqui MA, Qaseem SM, Mittal S, Iraqi AA, Rizvi SA. Role of magnetic resonance imaging in evaluation of tubercular spondylitis: pattern of disease in 100 patients with review of literature. JNMA J Nepal Med Assoc 2011;51:116-21. 